COMUNICAÇÃOCIENTÍFICA

\title{
CRUZAMENTOS EM CITROS: FREQÜÊNCIA E VIGOR DE HÍBRIDOS ${ }^{1}$
}

\author{
WALTER DOS SANTOS SOARES FILHO ${ }^{2}$, CARLOS ALBERTO DA SILVA LEDO ${ }^{2}$, MATHEUS PIRES QUINTELA ${ }^{3}$, \\ LORENNA ALVES MATTOS ${ }^{3}$, ORLANDO SAMPAIO PASSOS ${ }^{2}$, ANTÔNIO DA SILVA SOUZA ${ }^{2}$
}

RESUMO - Líder mundial, a citricultura brasileira compreende aproximadamente 270 milhões de plantas, distribuídas em mais de 900 mil ha. Vulnerável, devido ao uso excessivo do limoeiro 'Cravo' (Citrus limonia) na sustentação de seus pomares, exige urgência em um programa de diversificação de porta-enxertos. Nesse sentido, a Embrapa Mandioca e Fruticultura Tropical vem executando, no Recôncavo Baiano, um programa de hibridações visando a criar novas variedades, principalmente porta-enxertos, adaptadas a diferentes regiões geográficas do País, com ênfase no Nordeste. Considerando os caracteres altura da planta e diâmetro do caule, foram analisados 554 seedlings (plantas oriundas de sementes ou pés-francos) híbridos, dentro de 38 progênies obtidas de cruzamentos envolvendo Citrus e gêneros afins, com destaque para Poncirus. Os híbridos foram levados a campo entre agosto de 1995 e julho de 2000 , em conformidade com as épocas de suas obtenções. O citrangeiro (C. sinensis x P. trifoliata) 'Argentina' destacou-se como importante parental masculino na produção de híbridos. As tangerineiras 'Sunki (C. sunki) Comum', 'Sunki da Flórida', 'King' ( $C$. nobilis), 'Clementina ( $C$. clementina) de Nules' e 'Clementina Palazelli’ podem dar formação a progênies vigorosas. Seedlings híbridos de 'Sunki' e 'King' podem ser tão ou mais vigorosos que seedlings nucelares dessas tangerineiras.

Termos para indexação: Melhoramento genético, porta-enxerto, Citrus, Poncirus, Fortunella.

\section{CITRUS BREEDING: FREQUENCY AND VIGOR OF HYBRIDS}

\begin{abstract}
The Brazilian Citrus Industry is leading the world production, with about 270 million plants, in an area superior to 900 thousand ha. Provide that it is very vulnerable, due to the excessive use of 'Rangpur' lime (Citrus limonia) as rootstock; it demands urgency in a diversification program of rootstock varieties. Therefore, Embrapa Cassava\& Fruits is accomplishing a breeding program at Bahia State, Reconcavo Baiano, seeking the development of new varieties, mainly rootstocks, adapted to different geographic regions, with emphasis in the Northeast of Brazil. Considering the characters tree height and stem diameter, 554 seedlings hybrids from 38 progenies between Citrus and relatives, mainly Poncirus, were analyzed. The hybrids were planted between August 1995 and July 2000, according to their obtainable time. The 'Argentina' citrange ( $C$. sinensis x $P$. trifoliata $)$ stood out as important male parent for producing hybrids. The mandarins 'Common Sunki' (C. sunki), 'Florida Sunki', 'King' (C. nobilis), 'de Nules Clementine' (C. clementina) and 'Palazelli Clementine' can generate vigorous progenies. 'Sunki' and 'King' seedlings hybrids can be so or more vigorous than seedlings of nucellar origin of these mandarins.
\end{abstract}

Index terms: Genetic breeding, rootstock, Citrus, Poncirus, Fortunella.

\section{INTRODUÇÃO}

A citricultura brasileira destaca-se como a de maior expressão em nível mundial, com uma população de plantas da ordem de 270 milhões, distribuída, em todos os estados do País, em área superior a 900 mil ha (ANUÁRIO..., 2006), a maior parte, cerca de 800 mil ha, ocupada por laranjeiras-doces [Citrus sinensis (L.) Osbeck] (ANUÁRIO..., 2006; Neves \& Jank, 2006). Com 30\% da produção mundial de laranjeiras-doces e $59 \%$ da produção global de suco concentrado congelado de laranja, o sistema agroindustrial citrícola nacional movimenta aproximadamente $\mathrm{R} \$$ 9 bilhões anuais, gerando em torno de 400 mil empregos diretos e indiretos (Neves \& Jank, 2006).

A vulnerabilidade do parque citrícola brasileiro, todavia, é muito grande, devido ao predomínio do limoeiro 'Cravo' $(C$. limonia Osbeck) na sustentação dos pomares, tornando urgente um programa de diversificação de porta-enxertos. Essa condição de risco é crítica em razão do surgimento de diversas doenças que têm limitado a cultura dos citros, recentemente agravada pela ocorrência, no norte do Estado de São Paulo e sul do Triângulo Mineiro, da morte súbita dos citros em limoeiro 'Cravo', que hoje representa aproximadamente $85 \%$ dos porta-enxertos utilizados pela citricultura paulista (FUNDECITRUS, 2007).

Dada essa situação, em setembro de 1988, a Embrapa

\footnotetext{
'(Trabalho 139-06). Recebido em :12-09-2006. Aceito para publicação em : 18-04-2007. Trabalho apresentado no $3^{\circ}$ Congresso Brasileiro de Melhoramento de Plantas, realizado em Gramado no período de 09 a 12 de maio de 2005; pesquisa parcialmente financiada pelo CNPq e Fundecitrus.

${ }^{2}$ Pesquisadores da Embrapa Mandioca e Fruticultura Tropical, Caixa Postal 007, CEP 44380-000, Cruz das Almas, BA. O primeiro autor é bolsista do CNPq, e-mail: wsoares@cnpmf.embrapa.br

${ }^{3}$ Alunos do Curso de Engenharia Agronômica do Centro de Ciências Agrárias, Ambientais e Biológicas da Universidade Federal do Recôncavo da Bahia UFRB, bolsistas PIBIC/CNPq.
} 
Mandioca e Fruticultura Tropical iniciou um programa de hibridações, visando a produzir novas variedades, principalmente porta-enxertos, adaptadas a diferentes regiões geográficas de cultivo, com ênfase no Nordeste brasileiro, notadamente na Grande Unidade de Paisagem de Tabuleiros Costeiros, que ocupa quase toda a faixa costeira, desde o Amapá até o Rio de Janeiro, estendendo-se ao Vale do Rio Paraíba do Sul, no Estado de São Paulo (Rezende, 2000).

O presente trabalho traz informações relativas à freqüência e ao vigor de híbridos de citros obtidos a partir de cruzamentos controlados. Foi realizado no município de Cruz das Almas, Recôncavo Baiano, nas coordenadas geográficas $12^{\circ} 40^{\prime} 39^{\prime \prime}$ de latitude sul e $39^{\circ} 06^{\prime} 23^{\prime \prime}$ de longitude oeste, com altitude de $226 \mathrm{~m}$, em ambiente representativo da Grande Unidade de Paisagem de Tabuleiros Costeiros. O solo da área experimental apresenta-se bastante profundo, bem intemperizado, oriundo de sedimentos da formação Capim Grosso de baixa fertilidade química, classificado como Latossolo Amarelo distrófico típico, textura argilosa a moderada, ácido, com declividade entre $10 \%$ e $15 \%$, com horizontes subsuperficiais coesos, tendo as seguintes características físicas: areia total $444 \mathrm{~g} \mathrm{~kg}^{-1}$; silte $131 \mathrm{~g} \mathrm{~kg}^{-1}$; argila $425 \mathrm{~g} \mathrm{~kg}^{-1}$, e densidade do solo de $1,36 \mathrm{~kg} \mathrm{dm}^{-3}$ (Souza \& Souza, 2001). O clima da região, segundo a classificação de Köppen, é do tipo BSa, ou seja, com evapotranspiração potencial média anual maior do que a precipitação média anual, estação seca de verão e temperatura média superior a $22^{\circ} \mathrm{C}$ no mês mais quente do ano, estando a umidade relativa média anual em torno de $80 \%$ (D’Angiolella et al., 1998).

Um total de 38 progênies foi analisado, compreendendo 554 seedlings (plantas oriundas de sementes ou pés-francos) híbridos, resultantes de cruzamentos envolvendo os seguintes parentais (Tabelas 1 a 4): tangerineiras 'Clementina (C. clementina hort. ex Tanaka) de Nules' (TCLN), 'Clementina Comum' (TCLC), 'Clementina Palazelli' (TCLP), 'King' (C. nobilis Lour.) (TKG), 'Cleópatra' (C. reshni hort. ex Tanaka) (CLEO), 'Sunki [C. sunki (Hayata) hort. ex Tanaka] da Flórida' (TSKFL) e 'Sunki Comum' (TSKC), limoeiros 'Cravo' (LCR), 'Volkameriano (C. volkameriana V. Ten. \& Pasq.) Comum' (LVKC), 'Volkameriano Catânea 2' (LVKCT2) e 'Rugoso Mazoe' (C. jambhiri Lush.) (LRM), laranjeira 'Hamlin' (C. sinensis) (LHA), toranjeira 'Chandler' \{híbrido tipo toranja [C. maxima Burm. (Merr.)], obtido de cruzamento entre as toranjeiras 'Siamese Sweet' e 'Siamese Pink' (TCD), Poncirus trifoliata (L.) Raf. (TR) seleções 'Barnes' (TRBN), 'Beneke' (TRBK), Diplóide (TRDP), 'English' (TRENG), 'Flying Dragon' (TRFD) e 'Pomeroy' (TRPO), C. webberi Wester (CWEB), tangerineiratangeleiro 'Lee' [tangerineira 'Clementina' $\mathrm{x}$ tangeleiro 'Orlando' (pomeleiro 'Duncan' C. paradisi Macfad. x tangerineira 'Dancy' C. tangerina hort. ex Tanaka)] (LEE), citrangeiros (C. sinensis $\mathrm{x} P$. trifoliata) 'Troyer' (CTTR), 'Carrizo' (CTCZ), 'Rusk' (CTRK), 'Cunninghan' (CTCG), 'Argentina' (CTARG), 'Sanford'(CTSF), C13 (CTC13), C25 (CTC25) e C35 (CTC35), citrumeleiro (C.paradisi $\mathrm{x}$ P. trifoliata) 'Swingle'(CTSW), citrangequateiro 'Thomasville' [kumquat 'Oval' Fortunella margarita (Lour.) Swingle x citrangeiro 'Willits'] (CTQT), além de um híbrido de P. trifoliata denominado Hybrid (HYD).

Conforme convenção, nas indicações dos cruzamentos (hibridações), os parentais femininos foram os primeiros a serem apresentados. Por exemplo, no cruzamento TCLN x CTC35, TCLN foi o parental feminino (Tabela 1). Por tratar-se a tangerineira 'Clementina' de uma espécie monoembriônica, suas variedades, quando empregadas como parentais femininos em hibridações, somente originam indivíduos de natureza zigótica, razão pela qual não foram verificados seedlings nucelares (geneticamente idênticos à planta-mãe) dessa tangerineira (Tabelas $1 \mathrm{a}$ 4).

O número de polinizações controladas variou entre cruzamentos, em função da disponibilidade de flores na ocasião de suas realizações, tanto no tocante aos parentais masculinos, fornecedores de pólen, como femininos, fornecedores de flores no estádio de balão, ou seja, próximas da abertura das pétalas, ocasião em que suas anteras não se encontram deiscentes e seus estilo-estigmas mostram-se receptivos, úmidos; essas flores tiveram suas pétalas removidas, sendo depois emasculadas. A identificação de seedlings híbridos, obtidos desses cruzamentos controlados, de modo a separá-los daqueles de origem nucelar, deu-se mediante observações de suas características morfológicas, principalmente foliares, com destaque para a utilização da característica trifoliada, dominante, presente em $P$. trifoliata e em híbridos envolvendo essa espécie (Soost \& Cameron, 1975).

As mencionadas progênies, sob a condição de seedlings, conforme já referido, foram avaliadas com respeito ao seu vigor, considerando os caracteres altura da planta e diâmetro do caule, este medido a $20 \mathrm{~cm}$ de altura em relação à superfície do solo. Os híbridos foram levados a campo em diferentes momentos, de agosto de 1995 a julho de 2000, a depender das épocas de suas obtenções, em conformidade com um esquema de cruzamentos contínuos conduzido pelo Programa de Melhoramento Genético de Citros da Embrapa Mandioca e Fruticultura Tropical, estando, portanto, com idades variáveis, entre três e oito anos de permanência em campo, na ocasião das avaliações de vigor (dezembro de 2003). Embora tendo havido, em razão de um melhor aproveitamento de espaços disponíveis em campo, uma reocupação de covas em decorrência da morte de plantas, levando a uma interposição temporal entre as duas primeiras etapas de plantio (Tabelas $1 \mathrm{e} 2$ ), estas, à semelhança das demais etapas, ocuparam áreas distintas, sendo as avaliações realizadas de forma particular, em nível de cada uma delas.

Foram calculadas as estatísticas descritivas: média; intervalo de variação (IV), correspondente ao intervalo entre os valores mínimo e máximo observados; coeficiente de variação $(\mathrm{CV})$, dado por $\mathrm{CV}=($ desvio-padrão/média $) \times 100$, e coeficiente de assimetria de Pearson (AS), dado por AS $=[3 \times$ (média - mediana) $] /$ desvio-padrão.

Com base no total de híbridos levados a campo (Tabelas 1 
a 4), tem-se que os parentais masculinos que deram formação a maiores quantidades de híbridos, foram: TRBK (43 híbridos), CTCG (26), CTSF (26), CTARG (20) e CTC35 (17) em cruzamentos com TCLN (Tabelas 1 e 2); CTARG (18) em cruzamentos com TCLC (Tabela 2); CTARG (20) e CTTR (16) em cruzamentos com TSKFL (Tabela 2); CTARG (34), CTSW (33), TR x LCR (24) e CTQT (24) em cruzamentos com TSKC (Tabela 2); CTSW (51) em cruzamentos com LVKC (Tabelas 3 e 4). Esses resultados indicam que o citrangeiro 'Argentina' (CTARG) destacou-se como eficiente parental masculino nas hibridações controladas de que participou, sugerindo sua boa capacidade geral de combinação em cruzamentos, relativamente à obtenção de híbridos, tendo-se como referência o conjunto de parentais femininos utilizados.

Dentre os seedlings híbridos levados a campo no período de agosto de 1995 a junho de 1997 (Tabela 1), a progênie do cruzamento TKG x CTSW apresentou a maior média de altura da planta, da ordem de 3,0 $\mathrm{m}$, sendo este valor superior àquele constatado em seedling nucelar de TKG $(2,90 \mathrm{~m})$. O vigor relativamente elevado de híbridos desse cruzamento também pode ser verificado por meio do intervalo de variação, que indica valores de altura da planta entre 2,80 e 3,20 m. Essa progênie, além disso, apresentou baixo valor de CV $(6,48 \%)$ e coeficiente de assimetria negativo $(-0,46)$, no que concerne ao citado caráter, sugerindo, respectivamente, que esse cruzamento possibilita a obtenção de híbridos relativamente uniformes e vigorosos; o coeficiente de assimetria, quando negativo, significa que os valores do caráter ao qual se relaciona encontram-se concentrados acima da média observada para o mesmo, sendo essa tendência tanto mais expressiva quanto maior for o valor negativo obtido; situação contrária dá-se no tocante a valores positivos desse coeficiente (Bussab \& Morettin, 2002). Ainda referindo-se aos seedlings de origem zigótica plantados em campo entre agosto de 1995 e junho de 1997 (Tabela 1), considerando-se as hibridações envolvendo seleções de tangerineira 'Clementina', a progênie mais vigorosa foi a do cruzamento TCLN x HYD, com valores médios de altura da planta e diâmetro do caule de, respectivamente, $2,74 \mathrm{~m}$ e $8,27 \mathrm{~cm}$. Nessa etapa de plantio de híbridos, cabe acrescentar, constatou-se, em todas as progênies analisadas, que a variável diâmetro do caule apresentou valores de $\mathrm{CV}$ superiores aos daqueles verificados para altura da planta, situação esta que, em geral, se repetiu nas demais etapas de plantio de híbridos (Tabelas 2 a 4), indicando uma tendência de maior estabilidade fenotípica da variável altura da planta em relação à variável diâmetro do caule.

Relativamente aos híbridos plantados em campo entre julho de 1996 e agosto de 1998 (Tabela 2), as maiores médias para altura da planta foram obtidas nas progênies dos cruzamentos TSKC x CTSW (2,95 m), TCLN x TRDP (2,86 m), TSKFL x CTARG (2,76 m) e TSKFL x CTC13 (2,70 m). Dentre estas hibridações, cabe destacar o valor de CV relativamente baixo $(22,32 \%)$ e o coeficiente de assimetria negativo $(-0,48)$ constatados para TSKC x CTSW, que indicam, respectivamente, a obtenção de híbridos relativamente uniformes e com alturas de planta com tendência a serem superiores à média dessa variável. Observa-se, pelo IV, que em todas as progênies de cruzamentos com 'Sunki' houve seedlings híbridos cujas alturas de planta mostraram-se iguais ou superiores às de seedlings de origem nucelar dessa tangerineira, constatandose, nos conjuntos de híbridos dos cruzamentos TSKFL x CTARG e TSKFL x CTC13, valores extremos máximos superiores aos daqueles verificados em seedlings nucelares de TSKFL. Entre as progênies híbridas, a relativa ao cruzamento TSKC x CTSW apresentou, também, o maior diâmetro médio de caule $(8,97 \mathrm{~cm})$.

Entre os híbridos plantados em campo, em julho de 1999 (Tabela 3), verificou-se que a progênie do cruzamento TSKC $\mathrm{x}$ LHA manifestou as maiores médias de altura da planta e diâmetro do caule $(2,47 \mathrm{~m}$ e $5,45 \mathrm{~cm}$, respectivamente) e menores valores de CV (15,74\% e 15,86\%, respectivamente), indicando a obtenção de indivíduos vigorosos e relativamente uniformes. $\mathrm{O}$ valor positivo do coeficiente de assimetria verificado para altura da planta $(2,37)$ mostra uma tendência de prevalência de híbridos com valores inferiores aos da média desse caráter, enquanto no tocante ao diâmetro do caule o valor de AS foi praticamente nulo $(0,03)$, significando que os valores dessa variável distribuíram-se de forma simétrica em relação à média. Os intervalos de variação constatados para o citado cruzamento, referentes aos caracteres avaliados, indicam claramente que o vigor das progênies híbridas foi compatível com o vigor apresentado por seedlings nucelares do parental feminino (TSKC).

Quanto aos híbridos levados a campo em julho de 2000 (Tabela 4), os maiores destaques de vigor de planta relacionaramse ao cruzamento TSKFL x CWEB, com médias de altura da planta e diâmetro do caule de $2,23 \mathrm{~m}$ e $4,70 \mathrm{~cm}$, respectivamente. Considerando-se o caráter altura da planta, o $\mathrm{CV}$ relativamente baixo $(18,93 \%)$ e o coeficiente de assimetria negativo $(-0,40)$ obtidos indicam que esse cruzamento pode originar híbridos relativamente uniformes e com vigor de planta em geral superior à média.

Analisando os híbridos dos cruzamentos envolvendo o limoeiro 'Volkameriano Comum' (LVKC) como parental feminino, plantados em campo, em julho de 1999 (Tabela 3) e julho de 2000 (Tabela 4), não se verificou o mesmo comportamento observado nas progênies em que as tangerineiras 'Sunki' e 'Clementina' estiveram presentes, particularmente a primeira. Em todas as progênies [LVKC x (TSK x TRENG256), LVKC x CTSW e LVKC x LCR], as médias de altura da planta e diâmetro do caule dos híbridos obtidos foram inferiores às dos seedlings nucelares do citado limoeiro, indicando o vigor relativamente inferior manifestado nessas hibridações, muito embora a presença de híbridos com vigor compatível ao de alguns seedlings nucelares de 'Volkameriano', conforme se depreende pelos valores de IV relativos às progênies de LVKC $\mathrm{x}$ (TSK $\mathrm{x}$ TRENG256) e LVKC $\mathrm{x}$ CTSW, levadas a campo em 1999, que apresentaram indivíduos 
com vigor semelhante ao verificado em determinados seedlings nucelares de LVKC, segundo valores máximos de altura de planta e diâmetro de caule de, respectivamente, $2,2 \mathrm{~m} \mathrm{e} \mathrm{7,0} \mathrm{cm,} \mathrm{observados}$ no cruzamento LVKC x (TSK x TRENG256) e de 2,7 m e 6,0 cm, constatados no cruzamento LVKC x CTSW (Tabela 3 ).

Os comentários realizados mostraram que as tangerineiras 'Sunki Comum' e 'Sunki da Flórida', como parentais femininos, destacaram-se pela produção de progênies vigorosas; tendência semelhante foi identificada para a tangerineira 'King', embora, neste caso, os cruzamentos tenham sido restritos ao emprego do citrumeleiro 'Swingle' como parental masculino. Ao contrário, o limoeiro 'Volkameriano', quando empregado como parental feminino, originou progênies pouco vigorosas; foi evidente o maior vigor das progênies que tiveram como parental feminino a tangerineira 'Sunki' (TSKC x LHA e TSKFL x CWEB), em comparação com o que se deu com aquelas envolvendo o limoeiro 'Volkameriano', segundo se observa nas etapas de plantio relacionadas a 1999 (Tabela 3) e 2000 (Tabela 4).

A comparação entre seedlings híbridos e nucelares das tangerineiras 'Sunki' (seleções 'Comum' e 'da Flórida') e 'King', particularmente, mostrou que essas variedades podem produzir híbridos tão ou mais vigorosos que indivíduos de origem nucelar. Esses resultados confirmam os obtidos por Soares Filho et al. (1999), em avaliações de vigor de híbridos de 'Sunki'. A respeito de a possibilidade de seedlings híbridos serem tão ou mais vigorosos que seedlings nucelares dos parentais femininos envolvidos em determinados cruzamentos, Cameron \& Frost (1968) comentaram que hibridações realizadas por Walter Tennyson Swingle e Herbert John Webber, no início do século passado, demonstraram que muitos seedlings de citrangeiros podem ser mais vigorosos que os nucelares relacionados aos parentais femininos utilizados em sua obtenção; semelhantemente, cruzamentos entre citrangeiros e pomeleiros, bem como entre Fortunella (Swingle) e citrangeiros (citrangequateiros) resultaram em híbridos vigorosos; Webber e Swingle também obtiveram pequenas populações de C. maxima $\mathrm{x}$ P. trifoliata e de (C. sinensis x C. paradisi) x P. trifoliata que se mostraram muito vigorosas. Cabe destacar, aqui, a atual carência de resultados relacionados a programas de melhoramento genéticos de citros baseados em hibridações, conforme se verifica pelo restrito número de variedades híbridas presentes na citricultura mundial, onde a quase totalidade de variedades-copa procede de seleções de mutações espontâneas; dentre os porta-enxertos, apenas os citrangeiros 'Troyer' e 'Carrizo', obtidos em 1909, vêm sendo utilizados de forma relativamente expressiva (Navarro, 2005). Nesse contexto, menção especial também deve ser feita ao citrumeleiro 'Swingle', igualmente obtido no início do século passado, hoje presente, como porta-enxerto, em muitos pomares paulistas, em regiões de ocorrência da morte súbita dos citros, particularmente.

A relativa uniformidade observada entre híbridos de $C$. sunki é um indicativo de que essa espécie deve possuir níveis de homozigose mais elevados que o da maioria das espécies do gênero Citrus, qualificando-a como importante parental na obtenção de híbridos com potencial de uso como porta-enxerto, dada a previsibilidade de resultados relativamente maior dos cruzamentos com essa tangerineira. Estudos baseados em técnicas de bandeamento cromossômico, realizados por Cornélio et al. (2003), confirmam a condição de maior homozigosidade de $C$. sunki, em comparação com outras espécies de Citrus.

Considerando-se as condições ambientais do local em que o estudo foi conduzido e o conjunto de genótipos utilizados como parentais nos cruzamentos realizados, tem-se:

1- O citrangeiro 'Argentina' é um bom parental masculino relativamente à produção de híbridos.

2- As tangerineiras 'Sunki Comum', 'Sunki da Flórida', 'King', 'Clementina Comum', 'Clementina de Nules' e 'Clementina Palazelli' podem dar formação a progênies vigorosas.

3 - Seedlings híbridos de 'Sunki Comum', 'Sunki da Flórida' e 'King' podem ser tão ou mais vigorosos que seedlings nucelares dessas tangerineiras.

TABELA 1 - Avaliações de vigor [altura (m) e diâmetro do caule (cm; medido a $20 \mathrm{~cm}$ da superfície do solo)] de seedlings híbridos e nucelares plantados em campo, entre agosto de 1995 e junho de 1997. Programa de Melhoramento Genético de Citros da Embrapa Mandioca e Fruticultura Tropical. Cruz das Almas- BA. 2003.

\begin{tabular}{|c|c|c|c|c|c|c|c|c|c|}
\hline \multirow{2}{*}{ Cruzamentos } & \multirow{2}{*}{$\begin{array}{c}\mathrm{N}^{0} \text { de } \\
\text { híbridos }\end{array}$} & \multicolumn{4}{|c|}{ Altura } & \multicolumn{4}{|c|}{ Diâmetro do caule } \\
\hline & & Média & $I^{1}$ & $C V^{2}(\%)$ & $\mathbf{A S}^{3}$ & Média & IV $^{1}$ & $C V^{2}(\%)$ & $\mathbf{A S}^{3}$ \\
\hline $\mathrm{TCLN}^{4} \times \mathrm{CTC} 35^{5}$ & 17 & 2,42 & $1,20-3,50$ & 26,08 & $-0,12$ & 6,29 & $1,50-11,00$ & 46,28 & $-0,06$ \\
\hline TCLN x HYD ${ }^{6}$ & 11 & 2,74 & $2,00-3,50$ & 19,44 & 0,17 & 8,27 & $3,00-13,00$ & 38,83 & $-0,01$ \\
\hline $\mathrm{TCLN}^{\mathrm{x}} \mathrm{CTSW}^{7}$ & 09 & 2,56 & $1,60-3,50$ & 25,74 & $-0,18$ & 7,33 & $3,50-11,50$ & 43,39 & 0,16 \\
\hline TCLN $x\left(\operatorname{TRBN}^{8} \times\right.$ CLEO $\left.^{9}\right)$ & 06 & 2,47 & $1,50-3,20$ & 34,18 & $-0,32$ & 7,25 & $2,00-14,50$ & 62,26 & 0,66 \\
\hline $\mathrm{TCLC}^{10} \times \mathrm{CTC} 25^{11}$ & 06 & 2,06 & $1,30-3,00$ & 27,71 & $-0,07$ & 4,20 & $2,00-6,20$ & 44,94 & 0,01 \\
\hline $\mathrm{TKG}^{12} \times \mathrm{CTSW}$ & 06 & 3,03 & $2,80-3,20$ & 6,48 & $-0,46$ & 6,83 & $4,00-10,00$ & 34,22 & 0,16 \\
\hline Total de híbridos & 55 & & & & & & & & \\
\hline \multirow{2}{*}{ Nucelares } & $\mathrm{N}^{0}$ de & \multicolumn{4}{|c|}{ Altura } & \multicolumn{4}{|c|}{ Diâmetro do caule } \\
\hline & nucelares & Média & $I V^{1}$ & $\mathrm{CV}^{2}(\%)$ & $\mathbf{A S}^{3}$ & Média & $I^{1}$ & $C V^{2}(\%)$ & $\mathbf{A S}^{3}$ \\
\hline TKG & 01 & 2,90 & $2,90-2,90$ & - & - & 9,00 & $9,00-9,00$ & - & - \\
\hline
\end{tabular}

${ }^{1} \mathrm{IV}$ : intervalo de variação; ${ }^{2} \mathrm{CV}$ : coeficiente de variação; ${ }^{3} \mathrm{AS}$ : coeficiente de assimetria; ${ }^{4} \mathrm{TCLN}$ : tangerineira 'Clementina (Citrus clementina hort. ex Tanaka) de Nules'; ${ }^{5}$ CTC35: citrangeiro [C. sinensis (L.) Osbeck x Poncirus trifoliata (L.) Raf.] C35; ${ }^{6} \mathrm{HYD}$ : híbrido de P. trifoliata; ${ }^{7}$ CTSW: citrumeleiro (C. paradisi Macfad. x P. trifoliata) 'Swingle'; ${ }^{8} \mathrm{TRBN}$ : P. trifoliata 'Barnes'; ${ }^{9}$ CLEO: tangerineira 'Cleópatra' $(C$. reshni hort. ex Tanaka); ${ }^{10} \mathrm{TCLC}$ : tangerineira 'Clementina Comum'; ${ }^{11} \mathrm{CTC} 25$ : citrangeiro C25; ${ }^{12} \mathrm{TKG}$ : tangerineira 'King' (C. nobilis Lour.). 
TABELA 2 - Avaliações de vigor [altura (m) e diâmetro do caule (cm; medido a $20 \mathrm{~cm}$ da superfície do solo)] de seedlings híbridos e nucelares plantados em campo, entre julho de 1996 e agosto de 1998. Programa de Melhoramento Genético de Citros da Embrapa Mandioca e Fruticultura Tropical. Cruz das Almas- BA. 2003.

\begin{tabular}{|c|c|c|c|c|c|c|c|c|c|}
\hline \multirow{2}{*}{ Cruzamentos } & \multirow{2}{*}{$\begin{array}{c}\mathrm{N}^{0} \text { de } \\
\text { híbridos }\end{array}$} & \multicolumn{4}{|c|}{ Altura } & \multicolumn{4}{|c|}{ Diâmetro do caule } \\
\hline & & Média & $I^{1}$ & $C V^{2}(\%)$ & $\mathbf{A S}^{3}$ & Média & $I V^{1}$ & $C V^{2}(\%)$ & $\mathbf{A S}^{3}$ \\
\hline $\mathrm{TCLN}^{4} \times \mathrm{CTCG}^{5}$ & 26 & 1,74 & $0,60-3,30$ & 39,06 & 0,55 & 4,06 & $1,50-10,00$ & 50,06 & 1,36 \\
\hline TCLN x CTARG $^{6}$ & 20 & 1,74 & $0,70-2,60$ & 34,47 & $-0,20$ & 3,88 & $1,20-8,00$ & 42,31 & 0,52 \\
\hline $\mathrm{TCLN}^{\mathrm{x}} \mathrm{CTSF}^{7}$ & 26 & 1,62 & $0,60-2,90$ & 37,70 & 0,37 & 4,30 & $1,00-10,00$ & 48,52 & 1,05 \\
\hline TCLP $^{8} \times$ CTSW $^{9}$ & 15 & 2,24 & $1,80-3,00$ & 16,69 & 0,91 & 6,03 & $2,00-11,50$ & 42,08 & 0,58 \\
\hline TSKFL $^{10}$ x CTARG & 20 & 2,76 & $0,90-4,50$ & 30,23 & $-0,01$ & 6,88 & $1,50-11,50$ & 40,68 & 0,27 \\
\hline TSKC $^{11} \times$ CTARG & 34 & 2,61 & $0,65-4,10$ & 33,49 & $-0,42$ & 7,44 & $1,50-14,50$ & 47,96 & 0,11 \\
\hline TSKFL $x$ CTC $25^{12}$ & 07 & 1,84 & $0,80-3,20$ & 55,15 & 0,51 & 4,64 & $2,00-9,50$ & 59,54 & 0,99 \\
\hline TSKFL $x$ CTC $13^{13}$ & 14 & 2,70 & $1,70-4,20$ & 24,74 & 0,28 & 8,14 & $5,00-14,00$ & 33,15 & 1,17 \\
\hline TSKC $\mathrm{x}$ CTSW & 33 & 2,95 & $1,20-4,50$ & 22,32 & $-0,48$ & 8,97 & $3,00-15,50$ & 36,44 & 0,29 \\
\hline $\operatorname{TSKC} \times\left(\mathrm{TR}^{14} \times \operatorname{LCR}^{15}\right)$ & 24 & 2,64 & $1,10-3,70$ & 24,49 & $-0,94$ & 8,23 & $2,50-15,00$ & 45,89 & 0,27 \\
\hline TSKFL CTTR $^{16}$ & 16 & 2,66 & $1,50-3,30$ & 19,49 & $-1,15$ & 8,19 & $2,00-11,50$ & 32,53 & $-1,26$ \\
\hline TSKC CTRK ${ }^{17}$ & 06 & 2,49 & $1,60-4,00$ & 41,52 & 0,47 & 5,87 & $2,50-11,00$ & 67,64 & 0,65 \\
\hline TSKC $^{x}$ TRFD $^{18}$ & 07 & 2,03 & $1,20-3,00$ & 35,40 & 0,65 & 3,79 & $2,50-5,00$ & 22,69 & $-0,17$ \\
\hline $\mathrm{TSKC}_{\mathrm{x}} \mathrm{TRBK}^{19}$ & 05 & 2,41 & $1,75-3,10$ & 23,03 & 0,08 & 3,30 & $1,00-4,50$ & 40,94 & $-1,70$ \\
\hline TSKC $\mathrm{x}$ TRDP ${ }^{20}$ & 13 & 2,02 & $0,70-3,30$ & 39,92 & $-0,20$ & 4,35 & $1,00-10,00$ & 54,04 & 0,98 \\
\hline $\mathrm{TSKC}_{\mathrm{x}} \mathrm{CTQT}^{21}$ & 24 & 2,62 & $1,50-4,00$ & 26,35 & 0,31 & 7,33 & $1,90-13,00$ & 40,07 & 0,36 \\
\hline TCLC $^{22} \times$ CTARG & 18 & 1,57 & $0,50-3,50$ & 52,03 & 0,98 & 4,43 & $1,00-19,00$ & 89,23 & 3,21 \\
\hline TCLC $x$ TRPO ${ }^{23}$ & 13 & 1,86 & $0,65-2,40$ & 30,43 & $-0,92$ & 3,38 & $2,00-5,00$ & 30,25 & 0,29 \\
\hline TCLC x CTCG & 05 & 1,82 & $1,10-2,30$ & 31,66 & $-0,59$ & 3,80 & $1,50-8,00$ & 67,48 & 1,39 \\
\hline TCLN x TRBK & 43 & 2,10 & $0,70-3,50$ & 34,30 & 0,09 & 4,66 & $1,20-15,00$ & 66,56 & 1,56 \\
\hline TCLN x TRDP & 10 & 2,86 & $2,40-3,90$ & 15,17 & 1,53 & 8,32 & $5,00-19,00$ & 47,96 & 2,48 \\
\hline TCLN x CTC25 & 06 & 1,24 & $0,75-1,60$ & 25,90 & $-0,43$ & 2,75 & $2,00-3,00$ & 15,21 & $-1,54$ \\
\hline $\mathrm{LEE}^{24} \times \mathrm{CTC} 25$ & 05 & 1,20 & $0,70-1,60$ & 34,86 & $-0,51$ & 2,70 & $1,50-4,00$ & 36,10 & 0,08 \\
\hline $\mathrm{TCD}^{25} \times\left(\mathrm{CLEO}^{26} \times \mathrm{CTCZ}^{27} 226\right)$ & 10 & 1,97 & $1,25-2,60$ & 19,56 & $-0,23$ & 5,00 & $3,00-8,50$ & 34,50 & 0,97 \\
\hline Total de híbridos & 400 & & & & & & & & \\
\hline \multirow{2}{*}{ Nucelares } & $N^{0}$ de & \multicolumn{4}{|c|}{ Altura } & \multicolumn{4}{|c|}{ Diâmetro do caule } \\
\hline & nucelares & Média & $\mathbf{I V}^{1}$ & $\mathrm{CV}^{2}(\%)$ & $\mathbf{A S}^{3}$ & Média & $\mathbf{I V}^{1}$ & $\mathrm{CV}^{2}(\%)$ & $\mathbf{A S}^{3}$ \\
\hline TSKFL & 08 & 3,29 & $3,00-3,80$ & 8,98 & 0,66 & 9,95 & $7,00-15,70$ & 29,64 & 1,03 \\
\hline TSKC & 25 & 3,31 & $1,60-4,60$ & 18,52 & $-0,67$ & 10,74 & $5,50-17,40$ & 24,96 & 0,11 \\
\hline
\end{tabular}

${ }^{1} \mathrm{IV}$ : intervalo de variação; ${ }^{2} \mathrm{CV}$ : coeficiente de variação; ${ }^{3} \mathrm{AS}$ : coeficiente de assimetria; ${ }^{4} \mathrm{TCLN}$ : tangerineira 'Clementina (Citrus clementina hort. ex Tanaka) de Nules'; ${ }^{5}$ CTCG: citrangeiro [C. sinensis (L.) Osbeck x Poncirus trifoliata (L.) Raf.] 'Cunninghan'; ${ }^{6} \mathrm{CTARG:} \mathrm{citrangeiro} \mathrm{'Argentina';}{ }^{7} \mathrm{CTSF}$ : citrangeiro 'Sanford'; ${ }^{8} \mathrm{TCLP}$ : tangerineira 'Clementina Palazelli'; ${ }^{9} \mathrm{CTSW}$ : citrumeleiro (C. paradisi Macfad. x P. trifoliata) 'Swingle'; ${ }^{10} \mathrm{TSKFL}$ : tangerineira 'Sunki [C. sunki (Hayata) hort. ex Tanaka] da Flórida'; ${ }^{11} \mathrm{TSKC}$ : tangerineira 'Sunki Comum'; ${ }^{12} \mathrm{CTC} 25$ : citrangeiro C25; ${ }^{13} \mathrm{CTC} 13$ : citrangeiro C13; ${ }^{14} \mathrm{TR}$ : $P$ trifoliata ${ }^{15}$ LCR: limoeiro 'Cravo' (C. limonia Osbeck); ${ }^{16} \mathrm{CTTR}$ : citrangeiro 'Troyer'; ${ }^{17} \mathrm{CTRK}$ : citrangeiro 'Rusk'; ${ }^{18} \mathrm{TRFD}$ : $P$. trifoliata 'Flying Dragon'; ${ }^{19}$ TRBK: $P$. trifoliata 'Beneke'; ${ }^{20}$ TRDP: P. trifoliata Diplóide; ${ }^{21} \mathrm{CTQT}$ : citrangequateiro 'Thomasville' [kumquat 'Oval' Fortunella margarita (Lour.) Swingle x citrangeiro 'Willits']; ${ }^{22}$ TCLC: tangerineira 'Clementina Comum'; ${ }^{23} \mathrm{TRPO}$ : P. trifoliata 'Pomeroy'; ${ }^{24} \mathrm{LEE}$ : tangerineira 'Clementina' x tangeleiro 'Orlando' (pomeleiro 'Duncan' $C$. paradisi x tangerineira 'Dancy' $C$. tangerina hort. ex Tanaka); ${ }^{25} \mathrm{TCD}$ : toranjeira 'Chandler' \{híbrido tipo toranja [C. maxima Burm. (Merr.)], obtido de cruzamento entre as toranjeiras 'Siamese Sweet' e 'Siamese Pink' $\} ;{ }^{26}$ CLEO: tangerineira 'Cleópatra' $(C$. reshni hort. ex Tanaka); ${ }^{27} \mathrm{CTCZ}$ : citrangeiro 'Carrizo'.

TABELA 3 - Avaliações de vigor [altura (m) e diâmetro do caule (cm; medido a 20 cm da superfície do solo)] de seedlings híbridos e nucelares plantados em campo, em julho de 1999. Programa de Melhoramento Genético de Citros da Embrapa Mandioca e Fruticultura Tropical. Cruz das Almas- BA. 2003.

\begin{tabular}{|c|c|c|c|c|c|c|c|c|c|}
\hline \multirow{2}{*}{ Cruzamentos } & \multirow{2}{*}{$\begin{array}{c}\mathrm{N}^{0} \text { de } \\
\text { híbridos }\end{array}$} & \multicolumn{4}{|c|}{ Altura } & \multicolumn{4}{|c|}{ Diâmetro do caule } \\
\hline & & Média & $I^{1} V^{1}$ & $C V^{2}(\%)$ & $\mathbf{A \mathbf { S } ^ { 3 }}$ & Média & $I V^{1}$ & $\mathrm{CV}^{2}(\%)$ & $\mathbf{A S}^{3}$ \\
\hline LVKC $^{4} \times\left(\right.$ TSK $^{5} \times$ TRENG $\left.^{6} 256\right)$ & 13 & 1,53 & $0,65-2,20$ & 29,74 & $-0,28$ & 2,58 & $1,00-7,00$ & 65,25 & 1,63 \\
\hline $\mathrm{LVKC}^{\mathrm{x}} \mathrm{CTSW}^{7}$ & 44 & 1,48 & $0,40-2,70$ & 48,86 & 0,13 & 3,15 & $1,00-6,00$ & 41,11 & 0,23 \\
\hline $\mathrm{LVKC} \mathrm{LC \textrm {LR } ^ { 8 }}$ & 05 & 1,57 & $1,05-1,90$ & 19,96 & $-1,40$ & 4,10 & $2,50-6,00$ & 34,92 & 0,31 \\
\hline $\mathrm{TSKC}^{9} \times$ LHA $^{10}$ & 10 & 2,47 & $2,10-3,50$ & 15,74 & 2,37 & 5,45 & $4,00-7,00$ & 15,86 & 0,03 \\
\hline TCLC $^{11} \times$ LVKCT $^{12}$ & 09 & 2,16 & $1,80-2,70$ & 17,07 & 0,39 & 5,07 & $3,50-8,00$ & 36,54 & 0,96 \\
\hline Total de híbridos & 81 & & & & & & & & \\
\hline \multirow{2}{*}{ Nucelares } & $N^{0}$ de & \multicolumn{4}{|c|}{ Altura } & \multicolumn{4}{|c|}{ Diâmetro do caule } \\
\hline & nucelares & Média & IV $^{1}$ & $\mathrm{CV}^{2}(\%)$ & $\mathbf{A S}^{3}$ & Média & IV $^{1}$ & $\mathrm{CV}^{2}(\%)$ & $\mathbf{A S}^{3}$ \\
\hline$\overline{\text { LVKC }}$ & 7 & 2,61 & $2,20-3,30$ & 13,56 & 0,96 & 7,00 & $4,00-10,00$ & 20,41 & $-1,36$ \\
\hline TSKC & 3 & 2,48 & $1,85-3,20$ & 22,72 & $-0,17$ & 5,33 & $3,00-7,00$ & 39,12 & $-0,61$ \\
\hline
\end{tabular}

${ }^{1} \mathrm{IV}$ : intervalo de variação; ${ }^{2} \mathrm{CV}$ : coeficiente de variação; ${ }^{3} \mathrm{AS}$ : coeficiente de assimetria; ${ }^{4} \mathrm{LVKC}$ : limoeiro 'Volkameriano (Citrus volkameriana V. Ten. \& Pasq.) Comum'; ${ }^{5} \mathrm{TSK}$ : tangerineira 'Sunki' [C. sunki (Hayata) hort. ex Tanaka]; ${ }^{6}$ TRENG: Poncirus trifoliata (L.) Raf. 'English'; ${ }^{7} \mathrm{CTSW}$ : citrumeleiro (C. paradisi Macfad. x P. trifoliata) 'Swingle'; ${ }^{8} \mathrm{LCR}$ : limoeiro 'Cravo' (C. limonia Osbeck); ${ }^{9} \mathrm{TKSC}$ : tangerineira 'Sunki Comum'; ${ }^{10}$ LHA: laranjeira 'Hamlin' [C. sinensis (L.) Osbeck]; ${ }^{11}$ TCLC: tangerineira 'Clementina (C. clementina hort. ex Tanaka) Comum'; ${ }^{12}$ LVKCT2: limoeiro 'Volkameriano Catânea 2'. 
TABELA 4 - Avaliações de vigor [altura (m) e diâmetro do caule (cm; medido a $20 \mathrm{~cm}$ da superfície do solo)] de seedlings híbridos e nucelares plantados em campo, em julho de 2000. Programa de Melhoramento Genético de Citros da Embrapa Mandioca e Fruticultura Tropical. Cruz das Almas- BA. 2003.

\begin{tabular}{|c|c|c|c|c|c|c|c|c|c|}
\hline \multirow{2}{*}{ Cruzamentos } & \multirow{2}{*}{$\begin{array}{c}\mathrm{N}^{\mathbf{0}} \text { de } \\
\text { híbridos }\end{array}$} & \multicolumn{4}{|c|}{ Altura } & \multicolumn{4}{|c|}{ Diâmetro do caule } \\
\hline & & Média & $\mathbf{I V}^{1}$ & $\mathrm{CV}^{2}(\%)$ & $\mathbf{A S}^{3}$ & Média & $I^{1}$ & $C V^{2}(\%)$ & $\mathbf{A S}^{3}$ \\
\hline LVKC $^{4} \times$ CTSW $^{5}$ & 07 & 0,76 & $0,40-1,20$ & 44,21 & 0,50 & 1,47 & $1,00-2,00$ & 28,22 & 0,26 \\
\hline $\mathrm{TSKFL}^{6} \times \mathrm{CWEB}^{7}$ & 06 & 2,23 & $1,60-2,80$ & 18,93 & $-0,40$ & 4,70 & $2,50-7,50$ & 42,45 & 0,43 \\
\hline TCLC $^{8} \times$ LRM $^{9}$ & 05 & 1,15 & $0,90-1,45$ & 19,44 & 0,42 & 2,28 & $2,00-3,00$ & 18,19 & 1,92 \\
\hline Total de híbridos & 18 & & & & & & & & \\
\hline \multirow{2}{*}{ Nucelares } & $\mathrm{N}^{0}$ de & \multicolumn{4}{|c|}{ Altura } & \multicolumn{4}{|c|}{ Diâmetro do caule } \\
\hline & nucelares & Média & IV $^{1}$ & $C V^{2}(\%)$ & $\mathbf{A \mathbf { S } ^ { 3 }}$ & Média & $I^{1}$ & $C V^{2}(\%)$ & $\mathbf{A S}^{3}$ \\
\hline LVKC & 03 & 2,37 & $2,20-2,50$ & 6,45 & $-0,94$ & 4,43 & $3,00-5,50$ & 29,09 & $-1,18$ \\
\hline
\end{tabular}

${ }^{1} \mathrm{IV}$ : intervalo de variação; ${ }^{2} \mathrm{CV}$ : coeficiente de variação; ${ }^{3} \mathrm{AS}$ : coeficiente de assimetria; ${ }^{4} \mathrm{LVKC}$ : limoeiro 'Volkameriano (Citrus volkameriana V. Ten. \& Pasq.) Comum'; ${ }^{5} \mathrm{CTSW}$ : citrumeleiro [C. paradisi Macfad. x Poncirus trifoliata (L.) Raf.] 'Swingle'; ${ }^{6} \mathrm{TSKFL}$ : tangerineira 'Sunki [C. sunki (Hayata) hort. ex Tanaka] da Flórida'; ${ }^{7} \mathrm{CWEB}$ : C. webberi Wester; ${ }^{8} \mathrm{TCLC}$ : tangerineira 'Clementina (C. clementina hort. ex Tanaka) Comum'; 'LRM: limoeiro 'Rugoso Mazoe' (C. jambhiri Lush.).

\section{AGRADECIMENTOS}

Os autores expressam seus agradecimentos ao Srs. Antônio Sant'Ana da Silva e Getúlio de Souza Vieira, servidores do quadro de funcionários da Embrapa Mandioca e Fruticultura Tropical, pelas ações de manejo das populações de híbridos e realização de polinizações controladas, respectivamente, fundamentais à realização deste trabalho.

\section{REFERÊNCIAS}

ANUÁRIO brasileiro da fruticultura 2006=Brazilian Fruit Yearbook. Santa Cruz do Sul: Editora Gazeta Santa Cruz, 2006. $136 \mathrm{p}$.

BUSSAB, W. de O.; MORETTIN, P.A. Estatística básica. 5.ed. São Paulo: Ed. Saraiva, 2002. 526p.

CAMERON, J.W.; FROST, H.B. Genetics, breeding and nucellar embryony. In: REUTHER, W.; BATCHELOR, L.D.; WEBBER, H.J. (Ed.). The citrus industry. Berkeley: University of California, 1968. v. 2, p. 325-370.

CORNÉLIO, M.T.M.N.; FIGUEIRÔA, A.R.S.; SANTOS, K.G.B.; CARVALHO, R.; SOARES FILHO, W. dos S.; GUERRA, M. Chromosomal relationships among cultivars of Citrus reticulata Blanco, its hybrids and related species. Plant Systematics and Evolution, Viena, v. 240, p. 149-161, 2003.

D'ANGIOLELLA, G.L.B.; CASTRO NETO, M.T.; COELHO, E.F. Tendências climáticas para os tabuleiros costeiros da região de Cruz das Almas-BA. In: CONGRESSO BRASILEIRO DE ENGENHARIA AGRÍCOLA, 27., 1998, Poços de Caldas. Anais... Lavras: Sociedade Brasileira de Engenharia Agrícola, 1998. v. 1, p. 43-45.

FUNDECITRUS. Morte súbita dos citros - MSC. Disponível em: $<$ http://www.fundecitrus.com.br/doencas/ morte subita.html>. Acesso em: 13 fev. 2007.
NAVARRO, L. Necesidades y problemática de la mejora sanitaria y genética de los cítricos en España. Phytoma, Valencia, n.170, p.2-5, 2005.

NEVES, M.F.; JANK, M.S. (Coord.). Perspectivas da cadeia produtiva de laranja no Brasil: a Agenda 2015. Pensa Boletim Online, São Paulo, 2006. Disponível em: <http:// w w w.pe n s a o o g. b r / d o w n l o a d s / Agenda_Citrus_2015_PENSAICONE.pdf $>$. Acesso em: 13 fev. 2007.

REZENDE, J. de O. Solos coesos dos tabuleiros costeiros: limitações agrícolas e manejo. Salvador: SEAGRI - SPA, 2000. 117 p. (Série Estudos Agrícolas, 1).

SOARES FILHO, W. dos S.; MOREIRA, C. dos S.; CUNHA, M.A.P. da; CUNHA SOBRINHO, A.P. da; PASSOS, O.S.; MORAIS, L.S. Vigor híbrido em tangerina 'Sunki'. Pesquisa Agropecuária Brasileira, Brasília, v.34, n.5, p.903-909, 1999.

SOOST, R.K.; CAMERON, J.W. Citrus. In: JANICK, J.; MOORE, J. N. (Ed.). Advances in fruit breeding. West Lafaiette: Purdue University Press, 1975. p. 507-540.

SOUZA, L. da S.; SOUZA, L.D. Caracterização físico-hídrica de solos da área do Centro Nacional de Pesquisa de Mandioca e Fruticultura Tropical. Cruz das Almas: EMBRAPA-CNPMF, 2001. 56p. (Boletim de Pesquisa, 20). 